\title{
THE APPROXIMATION FUNCTION OF BRIDGE DECK VIBRATION DERIVED FROM THE MEASURED EIGENMODES
}

\author{
MilAn SOKOL ${ }^{a}$, MAGdAlÉNA KOMORNÍKOVÁ $^{b, *}$, TOMÁŠ BACIGÁL ${ }^{b}$, \\ MIGUEL X. RODRÍGUEZ ${ }^{c}$
}

\author{
${ }^{a}$ Department of Structural Mechanics, Faculty of Civil Engineering \\ Slovak University of Technology, Radlinského 11, SK-810 05 Bratislava, Slovakia \\ e-mail: milan.sokolestuba.sk \\ ${ }^{b}$ Department of Mathematics, Faculty of Civil Engineering \\ Slovak University of Technology, Radlinského 11, SK-810 05, Bratislava, Slovakia \\ e-mail: \{magdalena.komornikova, tomas.bacigal\} @stuba.sk \\ ${ }^{c}$ School of Engineering and Sciences, Campus Puebla \\ Monterrey Institute of Technology and Higher Education \\ Va Atlixcáyotl 2301, Reserva Territorial Atlixcáyotl, 72453, Puebla, Mexico \\ e-mail: rodriguez.miguel@itesm.mx
}

\begin{abstract}
This article deals with a method of how to acquire approximate displacement vibration functions. Input values are discrete, experimentally obtained mode shapes. A new improved approximation method based on the modal vibrations of the deck is derived using the least-squares method. An alternative approach to be employed in this paper is to approximate the displacement vibration function by a sum of sine functions whose periodicity is determined by spectral analysis adapted for non-uniformly sampled data and where the parameters of scale and phase are estimated as usual by the least-squares method. Moreover, this periodic component is supplemented by a cubic regression spline (fitted on its residuals) that captures individual displacements between piers. The statistical evaluation of the stiffness parameter is performed using more vertical modes obtained from experimental results. The previous method (Sokol and Flesch, 2005), which was derived for near the pier areas, has been enhanced to the whole length of the bridge. The experimental data describing the mode shapes are not appropriate for direct use. Especially the higher derivatives calculated from these data are very sensitive to data precision.
\end{abstract}

Keywords: eigenmode approximation, eigenmode derivatives, regression spline, spectral analysis, distributed parameter system.

\section{Introduction}

Vibration measurement on bridges has a long tradition. Today it is used primarily to verify the static and dynamic behaviour of a bridge immediately after its construction. Much more importance, however, is assigned to repeated measurements over the entire life of the bridge, which are used for structural health monitoring (SHM) (Farrar et al., 2001; Roshan et al., 2015; Wenzel, 2009). These measurements, although very carefully performed, give the results only at discrete

\footnotetext{
*Corresponding author
}

points, even though these results are often burdened with errors such as inaccuracies in the measuring equipment, a limited range and sensitivity of measuring devices, time synchronization problems with the results from the measurement of polygons with lengths of several hundred meters, the uncertainty of processing results, random effects of weather, temperature effects, and the like.

Modal analyses are popular and used for identifying not only common structures like bridges or buildings, but also for a wide range of different mechanical systems subjected to different specific conditions (Joannin and Thouverez, 2015; Li et al., 2016; Silva et al., 2015; Yang 
and Sultan, 2016).

Several methods for calculating vibration mode shape derivatives were discussed long time ago (Sutter et al., 1988; Yu et al., 1997). They include the finite-difference method, the modal method or the Nelsons method. In this case, mode shape derivatives were calculated with respect to various design variables like a specific mass or a specific dimension of the model. A review of calculating the derivatives of eigenvalues and eigenvectors associated with general (non-Hermitian) matrices is provided by Murthy and Haftka (1988). ZhangPing and JinWu (2007) presented a novel modal superposition method for calculating eigenvector derivatives. This method is applicable to various damped systems and closed-mode cases. Yi et al. (2012) presented a discrete wavelet transform (DWT) approach to denoise the measured signals. Also Baili et al. (2009), Leite et al. (2008) and many others used the wavelet transform for similar purposes.

An approximation of a linear transfer function model is proposed, based on dynamic properties represented by a frequency response, e.g., determined as a result of discrete-time identification, by Janiszowski (2014). The denoising performance of the DWT is achieved by several processing parameters, including the type of wavelet, the decomposition level, thresholding method, and threshold selection rules. Liu (2013) presented a new method for calculating derivatives of eigenvalues and eigenvectors of discrete structural systems focused on discrete structural systems which are characterized by its eigenpairs, i.e., eigenvalues and eigenvectors. Eigenpair derivatives have been used in many areas such as design sensitivity analysis and optimization, correlation and model updating, stability analysis control, etc.

But all those papers are concerned mostly with parametric sensitivity of eigenvalues and eigenvectors or intended to denoise data with quite a big portion of high frequency noise. In our paper the focus is on smoothing the inaccurate test data without significant noise by using an appropriate approximation function. The basic requirement is that the approximation functions should enable calculation of higher order derivatives for expressing physical quantities of the structure, like, e.g., internal forces, etc.

When processing the results of measurements we have to find, as precisely as possible, the function of the deflection of the bridge. What is more, we need to handle its other derivatives, which are, e.g., rotation (the first derivative) or curvature (the second derivative) (De Roeck et al., 2000), or the third derivative, which describes the changes in shear forces.

For this purpose, we need to smooth the measured data very carefully. Thus, we are looking for an approximation function which corresponds accurately to the measuring results and at the same time meets the other boundary conditions, such as, e.g., those where due to the pillar the third derivative at supports is not a continuous function.

In this article we deal with the issue of how to acquire approximate displacement vibration functions. Input values are the discrete experimentally obtained mode shapes. A new improved approximation method based on the modal vibrations of the deck is derived on the basis of the weighted least-squares method. The statistical evaluation of the stiffness parameter is done using more vertical modes obtained from experimental results.

The method based on the solution of vibration for distributed parameter systems seems to be appropriate for approximation because of its original physical meaning describing a vibration of the structure. One parameter already measured during the tests that can be used here is an eigenfrequency of vibration. An approximation using this method has been derived for near the pier areas (Sokol and Flesch, 2005), and in this paper it is enhanced to the whole length of the bridge.

An alternative method to be employed in this paper is to approximate the displacement vibration function by a sum of sine functions whose periodicity (in the first step) is determined by spectral analysis adapted for non-uniformly sampled data and (in the second step) the parameters of the scale and phase are estimated as usual where by least squares method. Moreover, this periodic component is supplemented by a cubic regression spline-fitted on its residuals (as a third step) - that captures individual displacements between piers.

The paper is organized as follows. Section 2 presents spectral analysis of non-uniformly sampled data, a brief overview of the theory of regression splines, approximation functions based on the solution of vibration for distributed parameter systems and an evaluation of the approximation function by analysing the shear wave velocities obtained from vibration of a bridge. In Section 3 some practical applications are presented. Section 4 is dedicated to comparing advantages of the presented techniques and presents future research directives. Finally, some concluding remarks are added.

\section{Methods}

Since the displacement vibration function can be decomposed into a global periodic component and locally defined residual components, we briefly describe methods first to detect the period, to model the periodic component and lastly to capture the shape of each individual segment (between two adjacent piers) by a single continuous function. 
An alternative method of approximation is based on the assumption of transverse vibration of a beam with distributed parameters.

\subsection{Spectral analysis of non-uniformly sampled} data. Consider a response variable $Y$ and a spatial explanatory variable $X$ with (zero-mean) realizations $y_{1}, \ldots, y_{n}$ measured at points $x_{1}, \ldots, x_{n}$, respectively. We assume that the data series consists of a finite number of sinusoidal components and of a random noise. Formally,

$$
y_{i}=\sum_{j=1}^{m} A_{j} \cos \left(w_{j} x_{i}+\varphi_{j}\right)+\varepsilon_{i}
$$

where $w_{j}=2 \pi / T_{j}$ signifies the angular frequency corresponding to a period $T, \varphi_{j}$ is the phase and $A_{j}$ the amplitude of the $j$-th component, while $\varepsilon$ is, as usual, a random term. The problem of finding the set of significant frequencies $w_{j}$ is the task of spectral analysis and the most popular approach consists in computing the so-called periodogram.

If (at the experimental stage) the points $x_{i}$ were set with a constant increment, we could use the classical Fourier transform-based periodogram,

$$
P_{F}(\omega)=\frac{1}{n^{2}}\left|\sum_{j=1}^{n} y_{j} e^{-\imath \omega x_{j}}\right|^{2},
$$

(with $\imath=\sqrt{-1}$ ) computed easily by the discrete Fourier transform for $n$ Fourier frequencies $w_{j}=2 \pi j / n, j=$ $0, \ldots, n-1$. The periodogram (1) can be obtained as a solution to the least-squares data fitting problem

$$
\begin{aligned}
P_{F}(\omega) & =|\hat{\beta}|^{2}, \\
\hat{\beta} & =\underset{\beta}{\arg \min } \sum_{j=1}^{n}\left|y_{j}-\beta e^{\imath \omega x_{j}}\right|^{2},
\end{aligned}
$$

rewritten (if $\beta$ is expressed in polar form, $\beta=|\beta| e^{\imath 2 \pi \varphi}$ ) in the form

$$
\begin{aligned}
\hat{\beta}=\arg \min _{\beta} \sum_{i=1}^{n}\left[y_{i}-|\beta| \cos \left(\omega x_{i}+\varphi\right)\right]^{2} & \\
& +|\beta|^{2} \sin ^{2}\left(\omega x_{i}+\varphi\right) .
\end{aligned}
$$

The first term represents the least-squares fit of a sinusoidal function with a possible physical meaning; however, the second term acts as a data-independent perturbation. Thus, since our data points $x_{i}$ are distributed unevenly, the periodogram $P_{F}$ is not equivalent to the least-squares fit of sinusoidal functions (see Vio et al., 2013).
A more satisfactory spectral estimate should be obtained by solving the following least-squares problem:

$$
\min _{A \in R, \varphi \in[0,2 \pi]} \sum_{i=1}^{n}\left[y_{i}-A \cos \left(\omega x_{i}+\varphi\right)\right]^{2},
$$

reparametrized through $a=A \cos \varphi$ and $b=-A \sin \varphi$ to

$$
\min _{a, b} \sum_{i=1}^{n}\left[y_{i}-a \cos \left(\omega x_{i}\right)-b \sin \left(\omega x_{i}\right)\right]^{2} .
$$

The solution is

$$
\left(\begin{array}{l}
\widehat{a} \\
\widehat{b}
\end{array}\right)=R^{-1} r
$$

where

$$
\begin{aligned}
R & =\sum_{i=1}^{n}\left(\begin{array}{l}
\cos \left(\omega x_{i}\right) \\
\sin \left(\omega x_{i}\right)
\end{array}\right)\left(\begin{array}{ll}
\cos \left(\omega x_{i}\right) & \sin \left(\omega x_{i}\right)
\end{array}\right) \\
r & =\sum_{i=1}^{n}\left(\begin{array}{l}
\cos \left(\omega x_{i}\right) \\
\sin \left(\omega x_{i}\right)
\end{array}\right) y_{i} .
\end{aligned}
$$

Then the corresponding least-squares periodogram is defined as

$$
\begin{aligned}
P_{L S}(\omega) & =\frac{1}{n} \sum_{i=1}^{n}\left(\left(\begin{array}{ll}
\widehat{a} & \widehat{b}
\end{array}\right)\left(\begin{array}{l}
\cos \left(\omega x_{i}\right) \\
\sin \left(\omega x_{i}\right)
\end{array}\right)\right)^{2}, \\
& =\left(\begin{array}{ll}
\widehat{a} & \widehat{b}
\end{array}\right) R\left(\begin{array}{l}
\widehat{a} \\
\widehat{b}
\end{array}\right), \\
& =r^{T} R^{-1} r .
\end{aligned}
$$

In general, there is no guarantee that $P_{L S}(\omega)$ is independent of $P_{L S}\left(\omega^{\prime}\right)$ whenever $\omega \neq \omega^{\prime}$ since by (2) a single sinusoidal function is fitted per time. As a consequence, it is difficult to determine a correct threshold for significant frequencies detection. One may consider the quantity

$$
L_{F A}=-\sigma_{n}^{2} \ln \left[1-(1-\alpha)^{1 / n_{\omega}}\right],
$$

called the level of false alarm, i.e., the level that due to the noise (with standard deviation $\sigma_{n}$ ) one or more peaks would exceed with a prefixed probability $\alpha$ when $n_{\omega}$ (independent) frequencies are inspected. Though $n_{\omega}$ is not known, the dependence of $L_{F A}$ on $n_{\omega}$ is weak. Thus in many situations $n_{\omega}=n / 2$ can be a reasonable choice, as pointed out by Scargle (1982). For a discussion on pros and cons of the least-squares periodogram as well as a well-established modification, the so-called Lomb-Scargle periodogram, can be found in the works of Stoica et al. (2009) and Vio et al. (2013).

2.2. Regression spline. In many engineering applications a linear regression is a favourite method 
to approximate some dominant trend in the measured data. Sometimes a polynomial regression is used to accommodate the changing speed of growth or decrease, or specifically even a trigonometric polynomial makes sense when the trend is part of a very clear cyclic nature of the measured phenomenon. Such models are popular for their plain interpretability and are often chosen even when their fit is questionable, if not openly poor. As a straightforward remedy, one can choose higher order polynomials or more periodic components, yet then the interpretability is lost.

However, if we have a deeper knowledge of the data generating process and can reliably identify points where a structural change happens, one way out of the black box approach above can be to partition the regression problem to pieces. Let us, again, consider a response variable $Y$ and an explanatory variable $X$ with realizations $y_{1}, \ldots, y_{n}$ measured at points $x_{1}, \ldots, x_{n}$, respectively, and a linear model

$$
\begin{aligned}
y_{i}= & \boldsymbol{\beta}_{1} \boldsymbol{b}_{1}\left(x_{i}\right)+\boldsymbol{\beta}_{2} \boldsymbol{b}_{2}\left(x_{i}\right) \\
& +\cdots+\boldsymbol{\beta}_{K-1} \boldsymbol{b}_{K-1}\left(x_{i}\right)+\varepsilon_{i}
\end{aligned}
$$

with basis vector functions $\boldsymbol{b}_{k}$, parameter vectors $\boldsymbol{\beta}_{k}$, $k=1, \ldots, K-1$, and random terms $\varepsilon_{i}, i=1, \ldots, n$. If we know points $c_{1}, \ldots, c_{K}\left(c_{1} \leq x_{i}<c_{K}\right)$ where the observed phenomenon changes essentially, we may define the basis functions as

$$
\boldsymbol{b}_{k}(x)=\left(1, x, x^{2}, \ldots, x^{p_{k}}\right) I\left(c_{k} \leq x<c_{k+1}\right),
$$

where $p_{k}$ is the order of a particular polynomial, and $I$ denotes the indicator function which evaluates to 1 if its argument is true and to 0 otherwise. Then the function (4) is called a piecewise polynomial and the change points $c_{1}, \ldots, c_{K}$ are known as knots. The parameter vectors $\boldsymbol{\beta}_{k}$ are of length $p_{k}+1$, and the fitting of such a piecewise function uses $\sum_{k=1}^{K-1} p_{k}$ degrees of freedom.

To impose the continuity or even smoothness of our polynomial at the knots, a set of constraints needs to be introduced into parameter estimation, which in turn reduces the overall complexity. This can be achieved in a "numeric way" by an optimization method or, more easily, by a reparametrization through the basis functions. We use the latter approach.

Let us restrict ourselves to cubic polynomials between all adjacent knots and request the continuity of the regression curve up to (and including) the second derivative. Then the model (4) is rewritten to

$$
\begin{aligned}
y_{i}= & \beta_{0}+\beta_{1} b_{1}\left(x_{i}\right)+\cdots+\beta_{3+1} b_{3+1}\left(x_{i}\right) \\
& +\beta_{3+K} b_{3+K}\left(x_{i}\right)+\varepsilon_{i}
\end{aligned}
$$

with basis functions for a cubic polynomial $b_{k}(x)=x^{k}$ for $k=1,2,3$, and the extra, truncated power basis functions

$$
b_{3+j}(x)=\max \left(x-c_{j}, 0\right)^{3}, \quad j=1, \ldots, K,
$$

where $K$ is again the number of knots, yet parameters and basis functions are scalar terms. Regression polynomials with continuity restrictions are called regression splines and the particular one represented by the model (6) which has continuous first and second derivatives at the knots is known as a cubic spline 1 Fitting a cubic spline with $K$ knots uses $K+4$ degrees of freedom. Enforcing one more order of continuity would lead to a global cubic polynomial. For further details on regression splines, we refer the reader to, e.g., Hastie et al. (2009, p. 485-585) or James et al. (2013).

In our application of modelling the bridge and an adjacent part of soil, the response variable $y$ represents the shape of the specific vibration mode, a distance from the first pier is used as the predictor $x$, and the knots are placed to the respective piers.

\subsection{Approximation functions based on the solution} of vibration for distributed parameter systems. The solution is divided into parts. One part can represent a particular span of the bridge or a part of it, where the main characteristics $\left(E I_{p}, \mu_{p}, \omega_{m}\right)$ remain constant.

The mode shape $m$ in the part $p$ can be approximated using the solution describing the transverse vibration of a beam (Clough and Penzien, 1993) (see Fig. 1). The mode approximation functions are

$$
\begin{aligned}
& p_{p, m}\left(x_{p}\right) \\
& =C_{1, p, m} \cosh \left(a_{p, m} x_{p}\right)+C_{2, p, m} \sinh \left(a_{p, m} x_{p}\right) \\
& \quad+C_{3, p, m} \cos \left(a_{p, m} x_{p}\right)+C_{4, p, m} \sin \left(a_{p, m} x_{p}\right),
\end{aligned}
$$

where

- $a_{p, m}=\sqrt[4]{\mu_{p} \omega_{m}^{2} / E I_{p}}$ is a vibration parameter for the $m$-th mode in the part $p$,

- $\mu_{p}$ stands for deck masses per unit length,

- $\omega_{m}$ is the experimental circular frequency of the $m$-th mode,

- $E I_{p}$ stands for bending stiffness,

- $C_{1, p, m}, C_{2, p, m}, \ldots, C_{4, p, m}$ signify integration constants of vibration functions.

Continuity conditions for two adjacent functions $p_{p, m}\left(x_{p}\right), p_{p+1, m}\left(x_{p+1}\right)$ can be written as

$$
\left.p_{p, m}^{(n)}\left(x_{p}\right)\right|_{x_{p}=l_{p}}=\left.p_{p+1, m}^{(n)}\left(x_{p+1}\right)\right|_{x_{p+1}=0}
$$

${ }^{1}$ It is claimed that cubic splines are the lowest-order spline for which the knot-discontinuity is not visible to the human eye. 


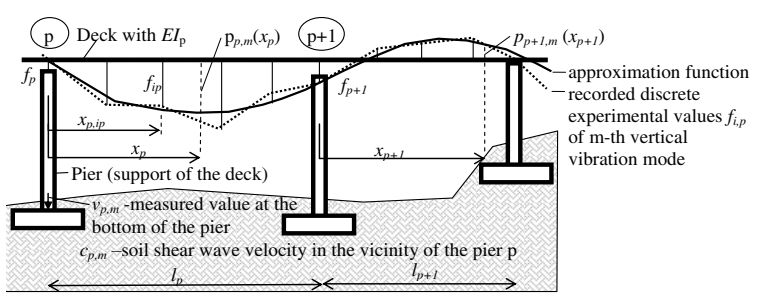

Fig. 1. Approximation function and the recorded vertical vibration mode.

for $n=0,1,2,3$, where $f^{(n)}(x)=\partial^{n} f(x) / \partial x^{n}$ denotes the $n$-th derivative and $l_{p}$ is the length of the part $p$. They ensure the continuity of the derivatives up to the third order. The continuity conditions $(8)$ for $n=0,1,2$ are required every time, while the condition for $n=3$ only in the case the boundary between two parts is located out of the support of the deck.

The overall approximation error $\tilde{E}$ is expressed by the sum of the squared differences between experimentally obtained values and the corresponding approximate values plus the squared differences between derivatives. The overall approximation error can be divided into two parts,

$$
\tilde{E}=\tilde{E}_{\text {out of boundary }}+\tilde{E}_{\text {at boundary }} .
$$

The first value represents the error calculated at all points lying out of the boundaries of adjacent parts,

$$
\tilde{E}_{\text {out of boundary }}=\sum_{p=1}^{N_{p}} \sum_{i_{p}=1}^{n_{p}}\left(f_{i_{p}}-p_{p, m}\left(x_{p, i_{p}}\right)\right)^{2},
$$

where

- $N_{p}$ is the number of parts,

- $n_{p}$ is the number of points in the part $p$, excluding points lying on the boundaries between adjacent parts,

- $f_{i_{p}}$ is the measured coordinate of the eigenmode at point $i_{p}$,

- $x_{p, i_{p}}$ is the local coordinate of the measured point $i_{p}$, and its calculated origin is located at the beginning of the part $p$.

The approximation error calculated at the adjacent boundaries is denoted as $\tilde{E}_{\text {at boundary. The contributions of }}$ higher derivatives are taken into account such that

$$
\begin{aligned}
& \tilde{E}_{\text {at boundary }} \\
& =\sum_{p=1}^{N_{p}-1} \alpha_{p}\left[\left(f_{p}-p_{p, m}\left(l_{p}\right)\right)^{2}+\left(f_{p}-p_{p+1, m}(0)\right)^{2}\right]
\end{aligned}
$$

$$
\begin{aligned}
& +\sum_{p=1}^{N_{p}-1} \beta_{p}\left(\frac{\partial p_{p, m}\left(l_{p}\right)}{\partial x_{p}}-\frac{\partial p_{p+1, m}(0)}{\partial x_{p+1}}\right)^{2} \\
& +\sum_{p=1}^{N_{p}-1} \gamma_{p}\left(\frac{\partial p_{p, m}^{2}\left(l_{p}\right)}{\partial x_{p}^{2}}-\frac{\partial p_{p+1, m}^{2}(0)}{\partial x_{p+1}^{2}}\right)^{2} \\
& +\sum_{p=1}^{N_{p}-1} \delta_{p}\left(\frac{\partial p_{p, m}^{3}\left(l_{p}\right)}{\partial x_{p}^{3}}-\frac{\partial p_{p+1, m}^{3}(0)}{\partial x_{p+1}^{3}}\right)^{2},
\end{aligned}
$$

where

- $\alpha_{p}$ is the weight of the mode shape continuity at the boundary between the parts $p$ and $p+1$,

- $\beta_{p}$ is the weight of the mode shape slope continuity at the boundary between the parts $p$ and $p+1$,

- $\gamma_{p}$ is the weight of the mode shape curvature continuity at the boundary between the parts $p$ and $p+1$,

- $\delta_{p}$ is the weight of the continuity of the third derivative of the mode shape at the boundary between the parts $p$ and $p+1$,

- $N_{p}-1$ is the number of boundaries between two adjacent parts,

- $f_{p}$ is the measured coordinate of the eigenmode at boundary $p$.

The values $\alpha_{p}, \beta_{p}, \gamma_{p}$ and $\delta_{p}$ can be assessed by parametric studies expressing the relative error of the approximation at the boundary between parts $p$ and $p+1$ (Sokol and Flesch, 2005). Larger $\beta_{p}, \gamma_{p}$ and $\delta_{p}$ values are expected since higher derivatives give values of a smaller magnitude. In (9), all error contributions are summed up. The influence of smaller magnitude values can be considered adequately in this way.

The condition for the extreme of the overall error function $\tilde{E}$ is

$$
\frac{\partial \tilde{E}\left(C_{1, p, m}, C_{2, p, m}, \ldots, C_{4, p, m}\right)}{\partial C_{j, p, m}}=0
$$

for $j=1, \ldots, 4$ and $p=1, \ldots, N_{p}$.

This leads to the system of $4 N_{p}$ equations

$$
\mathbf{A C}=\mathbf{R},
$$

where

$$
\mathbf{A}=\left(\begin{array}{cccc}
\mathbf{A}_{1,1} & \mathbf{0} & \cdots & \mathbf{0} \\
\mathbf{0} & \mathbf{A}_{2,2} & \cdots & \mathbf{0} \\
\vdots & \vdots & \ddots & \vdots \\
\mathbf{0} & \mathbf{0} & \cdots & \mathbf{A}_{N_{p}, N_{p}}
\end{array}\right)
$$


with

$$
\begin{aligned}
\mathbf{A}_{i . i}= & {\left[\begin{array}{ccc}
a_{1,1,(i), m} & \cdots & a_{1,8,(i), m} \\
\vdots & \ddots & \vdots \\
a_{4,1,(i), m} & \cdots & a_{4,8,(i), m}
\end{array}\right], } \\
\mathbf{C}= & \left(C_{1,(1), m}, \ldots, C_{4,(1), m}, \ldots,\right. \\
& \left.C_{1,\left(N_{p}\right), m}, \ldots, C_{4,\left(N_{p}\right), m}\right)^{T} \\
\mathbf{R}= & \left(r_{1,(1), m}, \ldots, r_{4,(1), m}, \ldots,\right. \\
& \left.r_{1,\left(N_{p}\right), m}, \ldots, r_{4,\left(N_{p}\right), m}\right)^{T}
\end{aligned}
$$

From this system of equations the coefficients of the approximation functions $C_{1,(1), m}, C_{2,(1), m}, C_{3,(1), m}$, $C_{4,(1), m}, \ldots, C_{1,\left(N_{p}\right), m}, C_{2,\left(N_{p}\right), m}, C_{3,\left(N_{p}\right), m}, C_{4,\left(N_{p}\right), m}$ can be calculated. The coefficients $a_{j, l,(p), m}$ and $r_{j,(p), m}, \quad j=1, \ldots, 4$, with $l=1, \ldots, 8$, and $p=1, \ldots, N_{p}$ in 13 are derived in such a manner that they can be calculated for an arbitrary number of parts $N_{p}$ and an arbitrary number of the measured points $n_{p}$ in the part $p$. The results are listed in Appendix.

The mode shape approximation functions $p_{p, m}\left(x_{p}\right)$ are found to smooth the experimental data well. The relative approximation error at the boundary between two parts $p$ and $p+1$ is defined by

$$
\tilde{e}_{p}=\sum_{n=0}^{3} \frac{\left|p_{p}^{(n)}\left(l_{p}\right)-p_{p+1}^{(n)}(0)\right|}{p_{p a}^{(n)}},
$$

where

$$
p_{p a}^{(n)}=\frac{1}{N} \sqrt{\sum_{p=1}^{N_{p}} \sum_{i_{p}=1}^{n_{p}}\left(p_{p, m}^{(n)}\left(x_{p, i_{p}}\right)\right)^{2}}
$$

with $N=\sum_{p=1}^{N_{p}} n_{p}$.

The unknown weight factors $\alpha_{p}, \beta_{p}, \gamma_{p}, \delta_{p}$, which give the minimum relative error $\tilde{e}_{p}$, can be found empirically in the following way.

The requirement of equality between approximation functions and experimental data in the position of the pier can be satisfied with the help of parameter $\alpha_{p}>$ 1 by definition. Then the parametrical calculation of the assessment of the relative approximation error $\tilde{e}_{p}$ is performed by varying parameters $\beta_{p}$ and $\gamma_{p}, \delta_{p}$ whose values can change across many orders of magnitude, e.g., one sets $\beta_{p}=10^{n}(n=-1,0,1, \ldots, 4)$ and $\gamma_{p}, \quad \delta_{p}=10^{n}(n=4,5, \ldots, 9)$. Keeping $\alpha_{p}$ fixed at the selected value, the relative approximation error function $\tilde{e}_{p}\left(\beta_{p}, \gamma_{p}\right)$ is then plotted in a diagram. In a usual case the parts are assumed between two supports of the deck, and then the continuity conditions for the third derivative must not be required and $\delta_{p}=0$. Assuming that the error should be small, say, no greater than $10 \%$, a combination of parameters $\alpha_{p}, \beta_{p}$ and $\gamma_{p}$ is identified from this diagram (Fig. 2). It remains to be checked that the approximation functions fit the experimental data in the vicinity of the pier. If $\beta_{p}$ and $\gamma_{p}$ are too large, the derivatives of the approximation functions may match nicely in the position of the pier but the fit to the data further away from the pier may deteriorate quickly. Thus it is desirable that the total error be divided evenly between the those computed from the fit to the data and contributions due to an imperfect matching of the approximation functions and their derivatives in the position of the pier. The values $\alpha_{p}=100, \beta_{p}=$ 150, $\gamma_{p}=80000, \delta_{p}=0$ are assumed in Section 3 .

2.4. Evaluation of the approximation function by analyzing the shear wave velocities obtained from the vibration of a bridge. Once we have smoothed approximation functions describing the vertical vibration in mode $m$, we can use them for analyzing the bridge pier vibration. Their third derivatives allow an estimate to be made of the amplitudes of the vertical deck reactions at the pier $p$ in the following way:

$$
\begin{aligned}
R_{p, m}= & -\left.E I_{p} \frac{\partial^{3} p_{p, m}\left(x_{p}\right)}{\partial x_{p}^{3}}\right|_{x_{p}=l_{p}} \\
& +\left.E I_{p+1} \frac{\partial^{3} p_{p+1, m}\left(x_{p+1}\right)}{\partial x_{p+1}^{3}}\right|_{x_{p+1}=0} .
\end{aligned}
$$

The procedure how to calculate shear wave velocities in subsoil in the vicinity of the vibrating bridge pier is described in detail by Sokol and Flesch (2005). For clarity, the basic steps of this calculation are repeated also here.

Shear wave velocities are assessed using several identified vertical mode shapes which were described by approximation functions derived above.

It is assumed that the dynamic soil properties depend also on frequency. The shear wave velocity and shear modulus are calculated using a method by Martinček $(1981$; 1994) which considers the vibration of a mass on an elastic half space.

Making use of the vertical deck reactions (15), the next step of investigation for each separate pier is relatively straightforward. The harmonic excitation forces of the pier-soil system in the $m$-th mode, caused by the vertical deck reaction, are assumed as follows:

$$
R_{p, m}(t)=R_{p, m} \sin \left(\omega_{m} t\right),
$$

where $\omega_{m}$ is the circular frequency of the vertical vibration of the pier, which is the same as the frequency of the deck measured experimentally. 


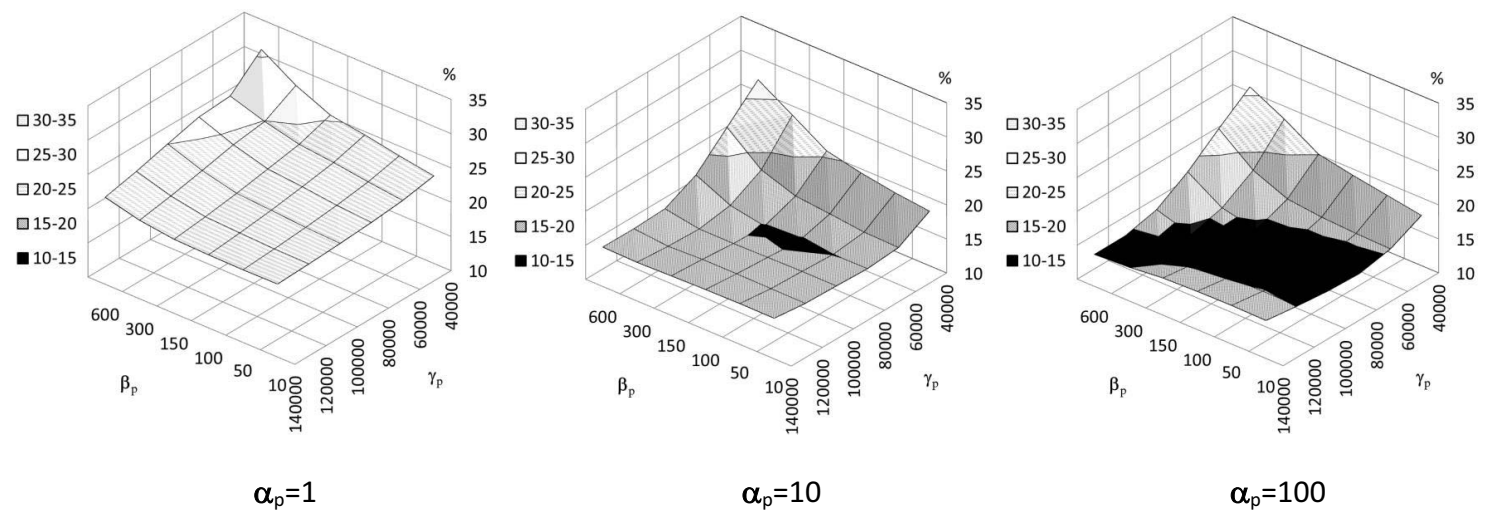

Fig. 2. Relative approximation error depending on $\alpha_{p}, \beta_{p}$ and $\gamma_{p}$.

The amplitudes of vertical deck reactions $R_{p, m}$ and the measured amplitudes of vertical pier bottom displacements $v_{p, m}$ at frequency $\omega_{m}$ are used to calculate the normalized mechanical impedance $\left|Z_{p, m}^{*}\right|$ (Martinček, 1981; Sokol and Flesch, 2005). Then the vertical pier-soil vibration is investigated as a single degree of freedom (SDOF) system expressed using complex stiffness. Knowing the mechanical impedance, an equivalent damping coefficient, a mass addition coefficient and the dimensionless frequency $\Omega_{p, m}$ can be calculated. Finally, the shear wave velocity can be obtained as

$$
c_{p, m}=\frac{\omega_{m} r_{p, e q v}}{\Omega_{p, m}},
$$

where $r_{p, e q v}$ is the radius of the equivalent circular pier foundation plate, with the same area as the rectangular pier foundation.

This soil characteristic-shear wave velocity-is compared with a geological survey available for a particular bridge. We used this for evaluation of the approximation function.

In most cases, experimental data are not precise enough to estimate the soil stiffness parameters using only one structural mode. Fortunately, more vertical modes are available and mean values can be calculated.

\section{Results}

Dynamic in situ testing using forced excitation was carried out in order to assess the dynamic properties of the Warth bridge in Austria. The bridge is built of seven pre-stressed concrete spans with a maximum span length of $67 \mathrm{~m}$. The total length of the structure is $459 \mathrm{~m}$. The maximum pier height is $39 \mathrm{~m}$. The deck is continuous with a box-girder cross section (see
Fig. 31. The results of the dynamic tests, which were performed by Arsenal Research, are summarized in the works of Flesch et al. (1999) as well as Sokol and Flesch (2005). The experimental modal analysis on the bridge was performed with the exclusion of traffic. Reference harmonic excitation was used and accelerations were recorded. By changing frequencies, the resonance vibrations could be recorded in more eigenmodes. The response variable realizations were recorded at every 10 locations along each particular span of the bridge.

All calculations related to splines as well as to spectral analysis were performed in the computer algebra system Wolfram Mathematica 10 (Wolfram Research, Inc., 2015), while the rest by the spreadsheet application Microsoft Excel 2013 (Microsoft, 2013).

The approximations, as well as the experimentally obtained vibration modes, are shown in Figs. 4 and 5 Third order derivatives of approximation functions are necessary for shear force estimation. In Fig. 6 there are introduced, e.g., higher derivatives for the sixth mode. This mode represents vertical vibration of all spans in the same direction, and so the second and third derivatives look after the bending moment and shear force diagrams due to gravity forces.

The analyzed shear wave velocities were compared with the reference analyses (Sokol and Flesch, 2005), calculated in Table 1. Weighted mean values are in Table 2. The displacements of the pier bottom (Table 3. were used as the weights.

This also proves that the smoothing of the

\footnotetext{
${ }^{2}$ Note that the results do not depend on the normalization of eigenmodes. Therefore the coordinates of the modes were used without normalization. They correspond to the values already published by Sokol and Flesch (2005). In addition, after processing the initially normalized eigenmodes the approximations will not be normalized any more.
} 


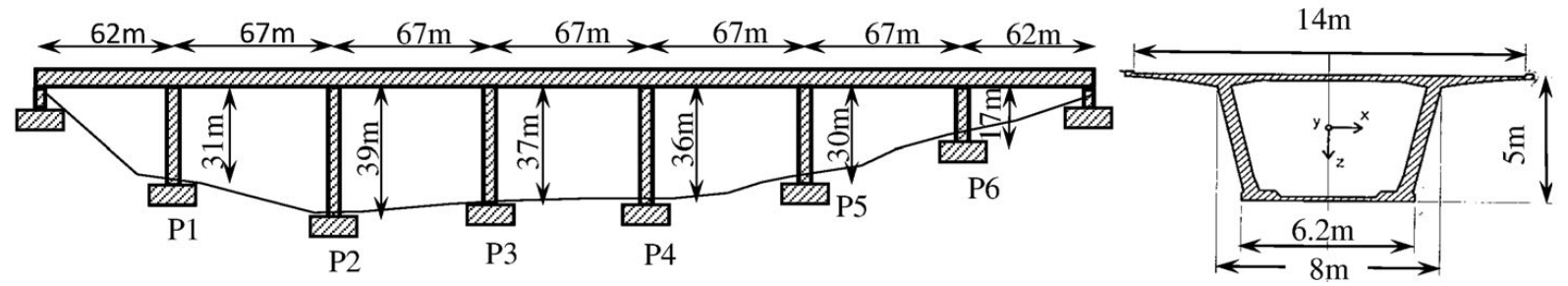

Fig. 3. Bridge structure (deck characteristics: $E I_{p}=7.94 \times 10^{11} \mathrm{~N} \mathrm{~m}^{2}, \mu_{p}=33420 \mathrm{~kg} / \mathrm{m}$.)

Table 1. Shear wave velocities (Sokol and Flesch, 2005).

\begin{tabular}{|c|c|c|c|c|c|c|c|c|c|}
\hline \multirow{2}{*}{$\begin{array}{c}c_{p, m} \\
{[\mathrm{~m} / \mathrm{s}]}\end{array}$} & \multicolumn{6}{|c|}{$\begin{array}{c}\mathrm{m} \\
\text { (mode) }\end{array}$} & \multirow{2}{*}{$\begin{array}{c}c_{\mathrm{avg}} \\
{[\mathrm{m} / \mathrm{s}]}\end{array}$} & \multirow{2}{*}{$\begin{array}{c}\sigma \\
{[\mathrm{m} / \mathrm{s}]}\end{array}$} & \multirow{2}{*}{$\begin{array}{l}\mathrm{CV} \\
\%\end{array}$} \\
\hline & 1 & 2 & 3 & 4 & 5 & 6 & & & \\
\hline 1 & 508 & 382 & 378 & 422 & 475 & 600 & 461 & 85 & 18 \\
\hline 2 & 511 & 203 & 440 & 344 & 171 & 391 & 343 & 134 & 39 \\
\hline 3 & 430 & 460 & 383 & 341 & 427 & 404 & 408 & 42 & 10 \\
\hline 4 & 63 & 267 & 356 & 380 & 329 & 338 & 289 & 117 & 40 \\
\hline 5 & 244 & 50 & 366 & 402 & 506 & 401 & 328 & 160 & 49 \\
\hline 6 & 249 & 717 & 317 & 359 & 526 & 529 & 449 & 173 & 39 \\
\hline
\end{tabular}

Table 2. Shear wave velocities (Sokol and Flesch, 2005): weighted mean values.

\begin{tabular}{|ccc|}
\hline$c_{\text {avg }}[\mathrm{m} / \mathrm{s}]$ & $\sigma[\mathrm{m} / \mathrm{s}]$ & $\mathrm{CV}$ \\
\hline \hline 467 & 21 & 5 \\
338 & 14 & 4 \\
391 & 8 & 2 \\
329 & 10 & 3 \\
362 & 18 & 5 \\
436 & 28 & 6 \\
\hline
\end{tabular}

experimentally obtained mode vibration values in the case of such a type of structure like this bridge is not easy and results are prone to errors, especially if higher derivatives are needed for the subsequent analysis for obtaining physical properties of soil shear velocities.

Results from approximation analyzes are given in Tables 4 and 5 (for the method using spline functions) and Tables 6 and 7 (for the method using distributed parameter system functions).

Comparing the results in Tables 4 and 5 as well as 6 and Table 7, it can be seen that in the case of calculating the results as weighted mean values (see Tables 5 and 7 ) the coefficient of variation $(\mathrm{CV})$ is less than $7 \%$, except one value in Table 5, for which the results were not reliable.

The comparison of the results is given in Figs. 7 (a) and (b). The results comply quite well to a geological investigation at the bridge site, where the outermost piers (nos. 1 and 6) are founded on rock soils, with the shear wave velocities expected to be higher than in the inner piers founded on alluvial deposits of sand and gravel.

\section{Discussion}

The method according to Sokol and Flesch (2005) provides reliable results close to the pier but not accurate results in midspan where two approximation functions have to be joined. These errors result from the assumption that the approximation functions are derived independently, each just around the nearest support. In our case the prescribed continuity conditions provide better continuous functions for the whole structure, and then the resulting functions accurately represent the true essence of the vibration of the structure. Even the continuity of higher order derivatives has been reached. These methods are suitable, e.g., for structural health monitoring (SHM), when repeated tests of the dynamic responses have to be compared and even small variations in results can lead to damage identification. In SHM of bridges it is necessary to know higher derivatives of eigenmodes in order to, e.g., assess stiffness using the direct method (Maeck and De Roeck, 1999a; 1999b) or for increasing the speed of iterations (Sokol et al., 2015).

Both methods described in the previous sections provide reliable results. The regression spline functions require the knots (at which no variation is allowed) to be 
Table 3. Measured amplitudes of vertical displacements of pier bottom parts (Sokol and Flesch, 2005).

\begin{tabular}{|c|cccccc|}
\hline & \multicolumn{6}{|c|}{$\mathrm{m}$ (mode) } \\
\cline { 2 - 7 }$p$ (pier) & 1 & 2 & 3 & 4 & 5 & 6 \\
\hline \hline 1 & 0.826 & 1.895 & 0.203 & 2.181 & 4.268 & 1.524 \\
2 & 0.724 & 2.369 & 1.218 & 15.657 & 2.044 & 6.007 \\
3 & 0.329 & 2.053 & 0.500 & 10.182 & 6.582 & 7.760 \\
4 & 0.908 & 4.465 & 1.546 & 7.303 & 7.299 & 12.455 \\
5 & 2.414 & 2.526 & 1.276 & 11.170 & 0.442 & 14.045 \\
6 & 6.614 & 0.638 & 0.847 & 1.696 & 2.987 & 10.882 \\
\hline
\end{tabular}

Table 4. Shear wave velocities: approximation using spline functions.

\begin{tabular}{|l|llllll|lll|}
\hline $\begin{array}{c}c_{p, m} \\
{[\mathrm{~m} / \mathrm{s}]}\end{array}$ & \multicolumn{7}{|c|}{$\begin{array}{c} \\
c_{\text {avg }} \\
{[\mathrm{m} / \mathrm{s}]}\end{array}$} & $\begin{array}{c}\sigma \\
{[\mathrm{m} / \mathrm{s}]}\end{array}$ & $\begin{array}{c}\mathrm{CV} \\
\%\end{array}$ \\
\hline$p($ pier) & 1 & 2 & 3 & 4 & 5 & 6 & & \\
\hline \hline 1 & 522 & 485 & 694 & 357 & 478 & 454 & 498 & 111 & 22 \\
2 & 608 & 385 & n.a. & 405 & 287 & 385 & 414 & 118 & 28 \\
3 & 769 & 147 & n.a. & 338 & 336 & 434 & 405 & 228 & 56 \\
4 & 327 & 260 & 370 & 374 & 78 & 374 & 297 & 116 & 39 \\
5 & 150 & 336 & 454 & 435 & n.a. & 399 & 355 & 123 & 35 \\
6 & 150 & 389 & 197 & 292 & 1052 & n.a. & 416 & 367 & 88 \\
\hline
\end{tabular}

Table 5. Weighted mean values of shear wave velocities: approximation using spline functions.

\begin{tabular}{|ccc|}
\hline$c_{\text {avg }}[\mathrm{m} / \mathrm{s}]$ & $\sigma[\mathrm{m} / \mathrm{s}]$ & $\mathrm{CV} \%$ \\
\hline \hline 459 & 19 & 4 \\
396 & 9 & 2 \\
349 & 17 & 5 \\
294 & 21 & 7 \\
384 & 14 & 4 \\
395 & 107 & 27 \\
\hline
\end{tabular}

identified in advance, yet by choosing truncated power basis functions we are able to selectively approximate displacement between piers by a smooth function and preserve the discontinuity of its third derivative. Note that the regression spline just fine-tunes the approximation by the bearing trigonometric polynomial function for which the frequency needs to be estimated from an unequally spaced data set. The least-squares periodogram serves that purpose well.

The distributed parameter approximation method is also reliable and robust. It is not sensitive to numerical errors and includes one of the main significant parameters of the dynamic response, namely, the measured circular frequency of vibration (7). This prevents rapid local changes in the approximation function due to the greater measurement uncertainties, which otherwise could lead to phantom changes in the curvature of the function representing the shape of vibration. This feature can be used to good advantage in SHM, when the results are sensitive to small variations in the compared displacements. Both methods were validated for a particular experiment, which is described in detail by Flesch et al. (1999) as well as Sokol and Flesch (2005). In this case, it was necessary to calculate third order derivatives. Then the shear wave velocities in subsoil were evaluated and compared with geological investigation. The regression spline approximation method yields results from which slightly higher values of shear wave velocities were obtained (Fig. 7) compared with those from distributed parameter approximation. The reason is that the continuity conditions are fulfilled precisely compared with the distributed parameter approximation method, where the continuity conditions at the boundaries of adjacent parts are fulfilled only to the extent required by parameters $\alpha_{p}, \beta_{p}, \gamma_{p}$ or $\delta_{p}$ (11). Accordingly, the curvatures (second and higher order derivatives) in the case of the first method are a little bit higher and the structure seems to be stiffer.

In Appendix we provide a general procedure for programming distributed parameter approximation functions representing a response of any type of linear structure like a bridge. An arbitrary number of measurement points and spans or even smaller parts of the bridge can be assumed. 
Table 6. Shear wave velocities: approximation using distributed parameter system functions.

\begin{tabular}{|l|cccccc|ccc|}
\hline $\begin{array}{c}c_{p, m} \\
{[\mathrm{~m} / \mathrm{s}]}\end{array}$ & \multicolumn{6}{|c|}{$\begin{array}{c} \\
c_{\text {avg }} \\
\text { (mode) }\end{array}$} & $\begin{array}{c}\sigma \\
{[\mathrm{m} / \mathrm{s}]}\end{array}$ & $\begin{array}{c}\mathrm{CV} \\
{[\mathrm{m} / \mathrm{s}]}\end{array}$ & $\%$ \\
\hline \hline$p$ (pier) & 1 & 2 & 3 & 4 & 5 & 6 & & \\
\hline \hline 1 & 455 & 391 & 487 & 444 & 464 & 548 & 465 & 52 & 11 \\
2 & 484 & 207 & 452 & 328 & 184 & 359 & 336 & 123 & 37 \\
3 & 492 & 485 & 364 & 337 & 409 & 374 & 410 & 65 & 16 \\
4 & n.a. & 282 & 368 & 360 & 324 & 314 & 330 & 35 & 11 \\
5 & 276 & 100 & 375 & 396 & 569 & 373 & 348 & 154 & 44 \\
6 & 237 & 693 & 295 & 386 & 480 & 483 & 429 & 162 & 38 \\
\hline
\end{tabular}
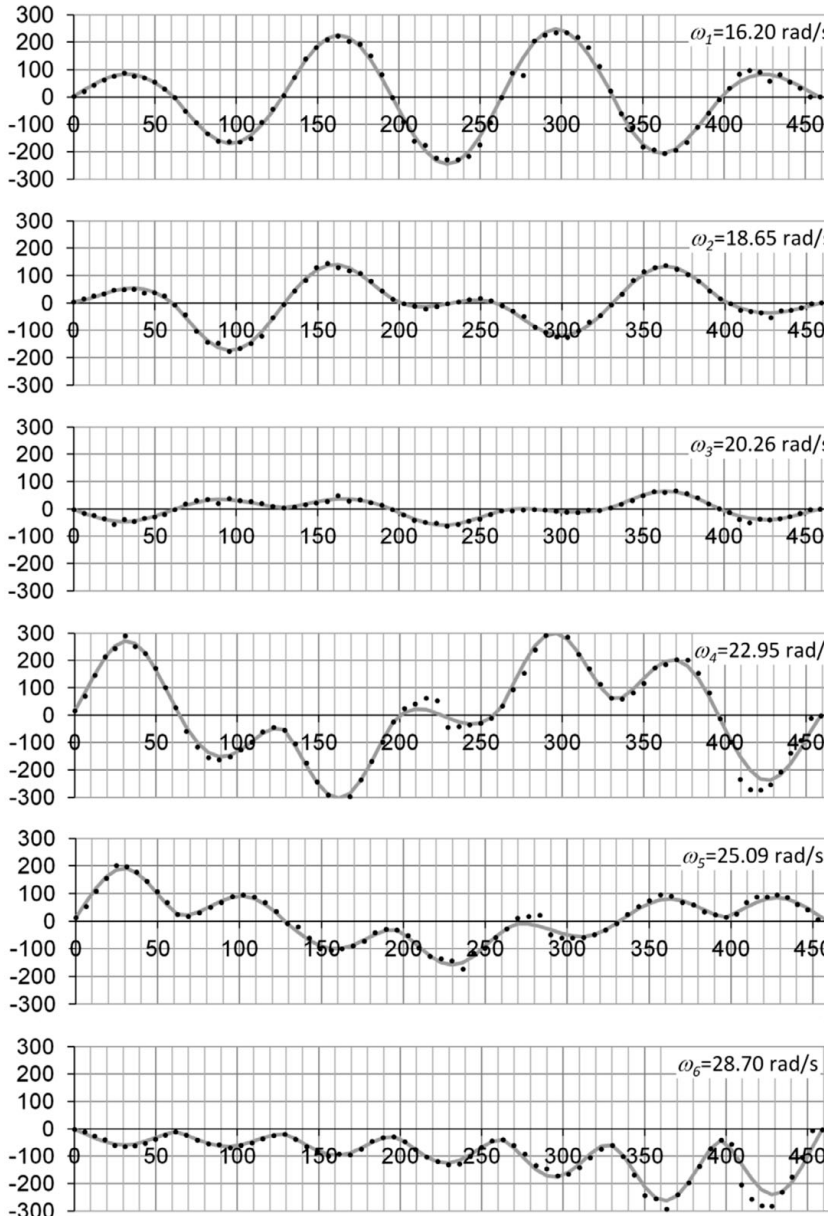
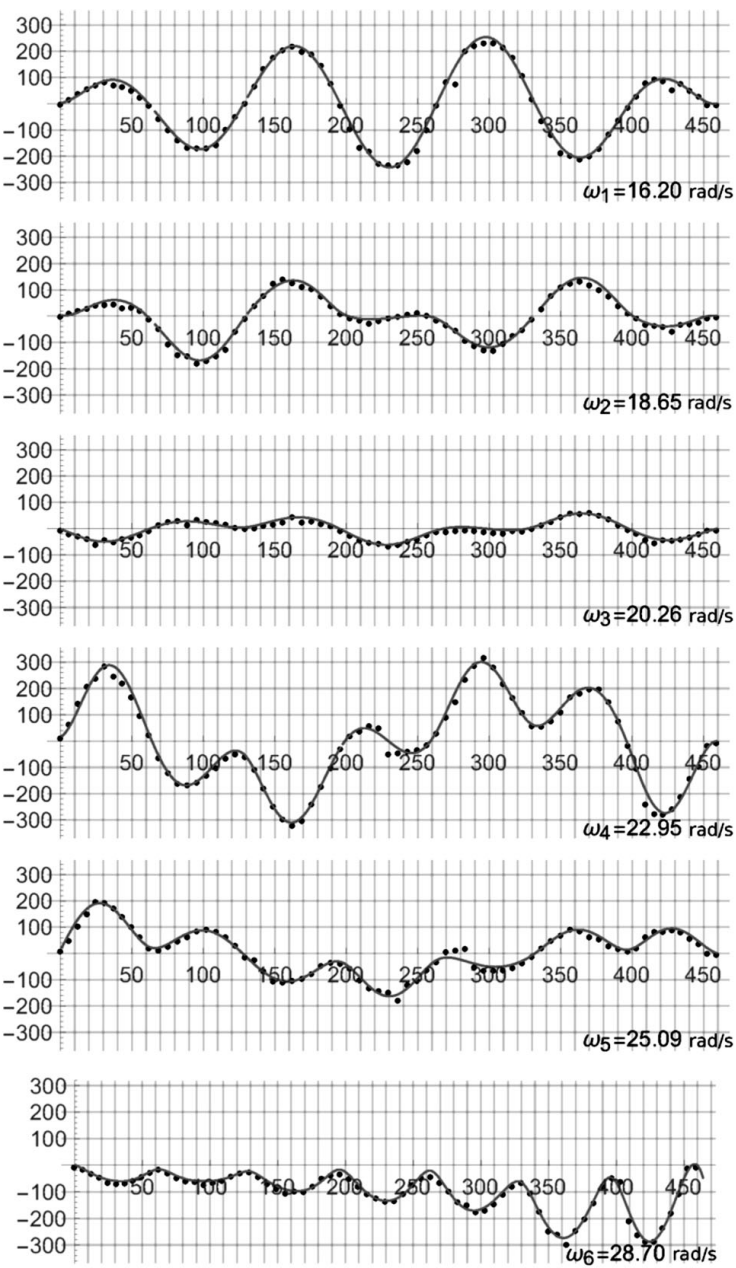

Fig. 4. Measured vibration modes and frequencies of a bridge and its smoothed approximation functions using a distributed parameter system (dotted line: test data, solid line: approximation function; the $x$-axis is expressed in meters, the $y$-axis is dimensionless).
Fig. 5. Measured vibration modes and frequencies of a bridge and its smoothed approximation functions using splines (dotted line: test data, solid line: approximation function; the $x$-axis is expressed in meters, the $y$-axis is dimensionless). 
Table 7. Weighted mean values of shear wave velocities approximation using distributed parameter system functions.

\begin{tabular}{|ccc|}
\hline$c_{\text {avg }}[\mathrm{m} / \mathrm{s}]$ & $\sigma[\mathrm{m} / \mathrm{s}]$ & $\mathrm{CV} \%$ \\
\hline \hline 459 & 14 & 3 \\
323 & 13 & 4 \\
378 & 8 & 2 \\
325 & 4 & 1 \\
355 & 15 & 4 \\
406 & 25 & 6 \\
\hline
\end{tabular}
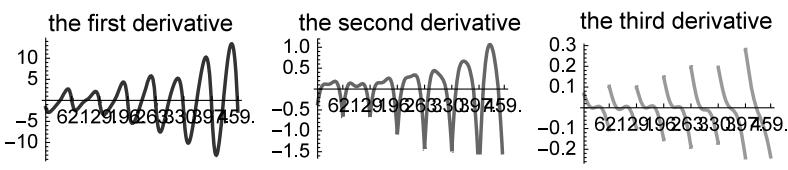

Fig. 6. Higher derivatives for mode no. 6 .

A further improvement of the results can be reached, if prior to the application of these methods apparent errors of measurement are analyzed so that inconsistencies can be eliminated as, e.g., in the case of eigenmode no. 4 between coordinates $220-230 \mathrm{~m}$ or eigenmode no. 5 between coordinates 280-300 $\mathrm{m}$ in Fig. 6. Of course, the proposed techniques smooth well also these error data, but the results lie somewhere between two apparent error displacements. A further improvement of the distributed parameter approximation method can be achieved by preparing a method for calculating appropriate values of coefficients $\alpha_{p}, \beta_{p}, \gamma_{p}$ or $\delta_{p}$ as they are described in (11) instead of guessing them from calculation of the relative error for many combinations.

\section{Conclusions}

The results produced by both approximation methods comply quite well with the geological investigation at the bridge site. Because the measured data are subjected to many sources of errors and they are not precise, an approximation method for smoothing vibration modes is necessary. The paper offers two methods: spline approximation and another one, using a distributed parameter system. Both are compared and can be used for similar purposes for a subsequent analysis. The described method of statistical evaluation of foundation (soil) parameters using vertical mode shapes identified from forced vibration experiments is appropriate for rough estimation of shear wave velocities and their changes along the bridge length.

The presented procedure of experimental data smoothing can also be used in other applications, where exact mathematical functions describing the vibration modes are needed, such as in system identification of
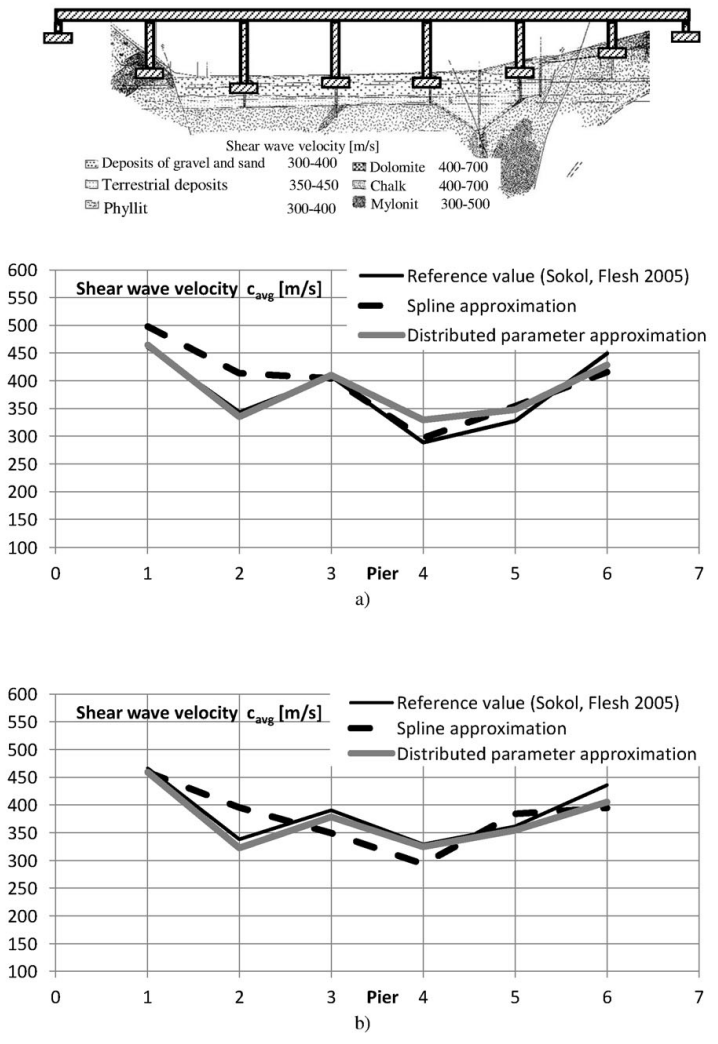

Fig. 7. Shear wave velocity $[\mathrm{m} / \mathrm{s}]$.

bridges or damage assessment using vibration tests. The presented method is useful especially in the cases where experimentally obtained data intended for further use are not accurate enough.

\section{Acknowledgment}

This research has been carried out within the framework of the research program no. APVV-0236-12 supported by the Slovak Research and Development Agency (SRDA), as well as the UVP research program no. ITMS 26240220084 supported by the Ministry of Education, Science, Research and Sport of the Slovak Republic. The financial and other support of the SRDA is gratefully acknowledged.

\section{References}

Baili, J., Lahouar, S., Hergli, M., Amimi, A. and Besbes, K. (2009). GPR signal de-noising by discrete wavelet transform, Nondestructive Testing and Evaluation International 42: 696-703.

Clough, R. and Penzien, J. (1993). Dynamics of Structures, McGraw-Hill, Singapore. 
De Roeck, G., Peeters, B. and Maeck, J. (2000). Dynamic monitoring of civil engineering structures, Computational Methods for Shells and Spatial Structures, IASS-IACM, Athens, Greece, pp. 1-24.

Farrar, C., Doebling, S. and Nix, D. (2001). Vibration-based structural damage identification, Philosophical Transactions of the Royal Society A359: 131-149.

Flesch, R., Stebernjak, B. and Freytag, B. (1999). System identification of bridge Warth/Austria, Structural Dynamics-EURODYN 99, Balkema, Rotterdam, pp. 813-818.

Hastie, T., Tibshirani, R. and Friedman, J. (2009). The Elements of Statistical Learning, Springer, New York, NY.

James, G., Witten, D., Hastie, T. and Tibshirani, R. (2013). An Introduction to Statistical Learning, 6th Edn., Springer, New York, NY.

Janiszowski, K.B. (2014). Approximation of a linear dynamic process model using the frequency approach and a non-quadratic measure of the model error, International Journal of Applied Mathematics and Computer Science 24(1): 99-109, DOI: 10.2478/amcs-2014-0008.

Joannin, C., Chouvion, B., Thouverez, F.and Mbaye, M.O.J. (2015). Nonlinear modal analysis of mistuned periodic structures subjected to dry friction, ASME Journal of Engineering for Gas Turbines and Power 138(7): 072504-072504-12.

Leite, F., Montagne, R., Corso, G., Vasconcelos, G. and Lucena, L. (2008). Optimal wavelet filter for suppression of coherent noise with an application to seismic data, Physica A 387: 1439-1445.

Li, P., Liu, C., Tian, Q., Hu, H. and Song, Y. (2016). Dynamics of a deployable mesh reflector of satellite antenna: Form-finding and modal analysis, ASME Journal of Computational and Nonlinear Dynamics 11(4): 041017-041017-12.

Liu, X. (2013). A new method for calculating derivatives of eigenvalues and eigenvectors or discrete structural systems, Journal of Sound and Vibration 332(7): 1859-1867.

Maeck, J. and De Roeck, G. (1999a). Detection of damage in civil engineering structures by direct stiffness derivation, 4th European Conference of Structural Dynamics, EURODYN 99, Prague, Czech Republic, pp. 485-490.

Maeck, J. and De Roeck, G. (1999b). Dynamic bending and torsion stiffness derivation from modal curvatures and torsion rates, Journal of Sound and Vibration 225(1): 153-170

Martinček, G. (1981). Soil investigation according to mechanical impedance method, Die Strasse 21(11): 374-379, (in German).
Martinček, G. (1994). Dynamics of Pavement Structures, E \& FN Spon., London.

Microsoft (2013). Microsoft Excel 2013, Redmond, WA.

Murthy, D. and Haftka, R.T. (1988). Derivatives of eigenvalues and eigenvectors of general complex matrix, International Journal for Numerical Methods in Engineering 26: $293-311$.

Roshan, P., Kumar, A., Tewatia, D. and Pal, S. (2015). Review paper on structural health monitoring: Its benefit and scope in India, Journal of Civil Engineering and Environmental Technology 2(2): 109-112.

Scargle, J.D. (1982). Studies in astronomical time series analysis. II: Statistical aspects of spectral analysis of unevenly spaced data, The Astrophysical Journal 263: 835-853.

Silva, T., Loja, L., Maia, N. and Barbosa, J. (2015). A hybrid procedure to identify the optimal stiffness coefficients of elastically restrained beams, International Journal of Applied Mathematics and Computer Science 25(2): 245-257, DOI: 10.1515/amcs-2015-0019.

Sokol, M., Aroch, R., Venglar, M., Fabry, M. and Zivner, T. (2015). Experience with structural damage identification of an experimental bridge model, Applied Mechanics and Materials 769: 192-199.

Sokol, M. and Flesch, R. (2005). Assessment of soil stiffness properties by dynamic tests on bridges, ASCE Journal of Bridge Engineering 10(1): 77-86.

Stoica, P., Li, J. and He, H. (2009). Spectral analysis of nonuniformly sampled data: A new approach versus the periodogram, IEEE Transactions on Signal Processing 57(3): 843-858.

Sutter, T., Gamarda, C.J., Walsh, J.L. and Adelman, H.M. (1988). Comparison of several methods for calculating vibration mode shape derivatives, AIAA Journal 26(12): 1506-1511.

Vio, R., Diaz-Trigo, M. and Andreani, P. (2013). Irregular time series in astronomy and the use of the Lomb-Scargle periodogram, Astronomy and Computing 1: 5-16.

Wenzel, H. (2009). Health Monitoring of Bridges, Wiley \& Sons, Chichester.

Wolfram Research, Inc. (2015). Mathematica 10.2, Champaign, IL.

Yang, S. and Sultan, C. (2016). Free vibration and modal analysis of a tensegrity-membrane system, 12th International Conference on Multibody Systems, Nonlinear Dynamics, and Control, Charlotte, NC, USA, Paper no. DETC2016-59292, pp. V006T09A023. 
Yi, T., Li, H. and Zhao, X. (2012). Noise smoothing for structural vibration test signals using an improved wavelet thresholding technique, Sensors 12: 11205-11220.

Yu, M., Liu, Z. and Wang, D. (1997). Comparison of several approximate modal methods for computing mode shape derivatives, Computers \& Structures 62(2): 381-393.

ZhangPing, L. and JinWu, X. (2007). Novel modal method for efficient calculation of complex eigenvector derivatives, AIAA Journal 45(6): 1406-1414.

Milan Sokol is a full professor of applied mechanics at the Slovak University of Technology (STU) in Bratislava, Slovakia. He graduated from the same institution in 1986, and obtained his $\mathrm{PhD}$ from the STU in 1994. He became an associate professor in 1998 and a full professor in 2009. He is a member of the editorial boards of the Slovak Journal of Civil Engineering and the Building Research Journal. Since 2011 he has been the chair of the Slovak Society for Mechanics. His fields of interest include non-linear statics and dynamics, design of bridges and buildings, structural analyses, structural health monitoring and biomechanics.

Magdaléna Komorníková graduated from Comenius University, Faculty of Mathematics and Physics, in 1973, and obtained her $\mathrm{PhD}$ at the same faculty in 1979. In 1976 she became a member of the Department of Kybernetics, and since 1990 she has been with the Department of Mathematics at the Slovak University of Technology Bratislava. She became an associate professor in 1985 and a full professor in 2002. Her fields of interests include measure theory, uncertainty modelling, copulas, time series analysis, aggregation and related operators, and engineering processes modelling.

Tomáš Bacigál graduated in 2003 from the Slovak University of Technology in Bratislava, Faculty of Civil Engineering, where he also obtained his $\mathrm{PhD}$ degree in 2007. He was then employed as an assistant professor by the Department of Mathematics and Constructive Geometry, and has worked there ever since. His fields of interests include multivariate modelling with copulas, time series analysis, data mining and statistical learning with applications in engineering.
Miguel X. Rodríguez is a full professor of engineering mechanics at Tecnológico de Monterrey, Puebla, Mexico. He obtained his PhD from the University of Wales, Swansea, in 2002. He was a senior research assistant from 2003 up to 2004 at Swansea. In 2004 he joined Tecnológico de Monterrey. He was given the title of National Researcher I by the Mexican Council for Science and Technology in 2006. His fields of interests include numerical methods in engineering, engineering education, innovation in education and social media in education, mechanics of materials, finite element method and structural dynamics.

\section{Appendix \\ Evaluation of the coefficients $a_{j, l, m}$ and $\boldsymbol{r}_{j, m}$}

Here $n_{p}$ is the number of the assumed measured points in the part $p$ excluding boundary points at the beginning and at the end of the part $p, x_{p, i_{p}}$ is the coordinate of the measured point $i_{p}$ (see Fig. 11). The derived results of coefficients $a_{j, l,(p), m}, j=1, \ldots, 4$, with $l=1, \ldots, 8$, and $p=1, \ldots, N_{p}$ along with $r_{j,(p), m} j=1, \ldots, 4$, and $p=1,2, \ldots, N_{p}$ are listed in Tables A1-A5.

We adopt the following notation:

$$
\begin{gathered}
a_{p, m}=\sqrt[4]{\frac{\mu_{p} \omega_{m}^{2}}{E I_{p}}}, \quad a_{p+1, m}=\sqrt[4]{\frac{\mu_{p+1} \omega_{m}^{2}}{E I_{p+1}}}, \\
A=a_{p, m}^{2}, \quad B=a_{p, m}^{4}, \quad G=a_{p, m}^{6}, \\
C h 0=\cosh \left(a_{p, m} 0\right)=1, \quad C h 1=\cosh \left(a_{p, m} l_{p}\right), \\
S h 0=\sinh \left(a_{p, m} 0\right)=0, \quad S h 1=\sinh \left(a_{p, m} l_{p}\right), \\
C 0=\cos \left(a_{p, m} 0\right)=1, \quad C 1=\cos \left(a_{p, m} l_{p}\right), \\
S 0=\sin \left(a_{p, m} 0\right)=0, \quad S 1=\sin \left(a_{p, m} l_{p}\right) .
\end{gathered}
$$


Table A1. Coefficients $r_{j,(p), m}, j=1, \ldots, 4$, and $p=1, \ldots, N_{p}$.

\begin{tabular}{|l|l|}
\hline Coefficient & \\
\hline \hline \multicolumn{2}{|l|}{ If $p<N_{p}$ then } \\
$r_{1,(p), m}$ & $\alpha_{p}\left(f_{p} C h 0+f_{p+1} C h 1\right)+\sum_{i_{p}=1}^{N_{p}} f_{i_{p}} \cosh \left(a_{p, m}, x_{p, i_{p}}\right)$ \\
$r_{2,(p), m}$ & $\alpha_{p}\left(f_{p} S h 0+f_{p+1} S h 1\right)+\sum_{i_{p}=1}^{N_{p}} f_{i_{p}} \sinh \left(a_{p, m}, x_{p, i_{p}}\right)$ \\
$r_{3,(p), m}$ & $\alpha_{p}\left(f_{p} C 0+f_{p+1} C 1\right)+\sum_{i_{p}=1}^{N_{p}} f_{i_{p}} \cos \left(a_{p, m}, x_{p, i_{p}}\right)$ \\
$r_{4,(p), m}$ & $\alpha_{p}\left(f_{p} S 0+f_{p+1} S 1\right)+\sum_{i_{p}=1}^{N_{p}} f_{i_{p}} \sin \left(a_{p, m}, x_{p, i_{p}}\right)$ \\
otherwise & \\
$r_{1,(p), m}$ & $\alpha_{p} f_{p} C h 1+\sum_{i_{p}}^{N_{p}} f_{i_{p}} \cosh \left(a_{p, m}, x_{p, i_{p}}\right)$ \\
$r_{2,(p), m}$ & $\alpha_{p} f_{p} S h 1+\sum_{i_{p}=1}^{N_{p}} f_{i_{p}} \sinh \left(a_{p, m}, x_{p, i_{p}}\right)$ \\
$r_{3,(p), m}$ & $\alpha_{p} f_{p} C 1+\sum_{i_{p}=1}^{N_{p}} f_{i_{p}} \cos \left(a_{p, m}, x_{p, i_{p}}\right)$ \\
$r_{4,(p), m}$ & $\alpha_{p} f_{p} S 1+\sum_{i_{p}=1}^{N_{p}} f_{i_{p}} \sin \left(a_{p, m}, x_{p, i_{p}}\right)$ \\
\hline
\end{tabular}

Table A2. Coefficients $a_{1, l,(p), m}, l=1, \ldots, 8$, and $p=1, \ldots, N_{p}$.

\begin{tabular}{|c|c|}
\hline Coefficient & \\
\hline$a_{1,1,(p), m}$ & $\begin{array}{l}\alpha_{p}\left(C h 0^{2}+C h 1^{2}\right)+\beta_{p} A S h 1^{2}+\gamma_{p} B C h 1^{2}+\delta_{p} G S h 1^{2} \\
+\sum_{i_{p}=1}^{N_{p}}\left(\cosh \left(a_{p, m}, x_{p, i_{p}}\right)\right)^{2}\end{array}$ \\
\hline$a_{1,2,(p), m}$ & $\begin{array}{l}\alpha_{p}(C h 0 S h 0+C h 1 S h 1)+\beta_{p} A C h 1 S h 1+\gamma_{p} B C h 1^{2}+\delta_{p} G S h 1 C h 1 \\
+\sum_{i_{p}=1}^{N_{p}}\left(\sinh \left(a_{p, m}, x_{p, i_{p}}\right) \cosh \left(a_{p, m}, x_{p, i_{p}}\right)\right)\end{array}$ \\
\hline$a_{1,3,(p), m}$ & $\begin{array}{l}\alpha_{p}(C h 0 C 0+C h 1 C 1)-\beta_{p} A S h 1 S 1-\gamma_{p} B C h 1 C 1+\delta_{p} G S h 1 S 1 \\
+\sum_{i_{p}=1}^{N_{p}}\left(\cosh \left(a_{p, m}, x_{p, i_{p}}\right) \cos \left(a_{p, m}, x_{p, i_{p}}\right)\right)\end{array}$ \\
\hline$a_{1,4,(p), m}$ & $\begin{array}{l}\alpha_{p}(C h 0 S 0+C h 1 S 1)+\beta_{p} A S h 1 C 1-\gamma_{p} B C h 1 S 1-\delta_{p} G S h 1 C 1 \\
+\sum_{i_{p}=1}^{N_{p}}\left(\cosh \left(a_{p, m}, x_{p, i_{p}}\right) \sin \left(a_{p, m}, x_{p, i_{p}}\right)\right)\end{array}$ \\
\hline \multicolumn{2}{|c|}{ If $p<N_{p}$ then } \\
\hline$a_{1,5,(p), m}$ & $-\gamma_{p} a_{p, m}^{2} a_{p+1, m}^{2} C h 1$ \\
\hline$a_{1,6,(p), m}$ & $-\beta_{p} a_{p, m} a_{p+1, m}-\delta_{p} a_{p, m}^{3} a_{p+1, m}^{3} S h 1$ \\
\hline$a_{1,7,(p), m}$ & $+\gamma_{p} a_{p, m}^{2} a_{p+1, m}^{2} C h 1$ \\
\hline$a_{1,8,(p), m}$ & $-\beta_{p} a_{p, m} a_{p+1, m}+\delta_{p} a_{p, m}^{3} a_{p+1, m}^{3} S h 1$ \\
\hline
\end{tabular}


Table A3. Coefficients $a_{2, l,(p), m}, l=1, \ldots, 8$, and $p=1, \ldots, N_{p}$.

\begin{tabular}{|c|c|}
\hline Coefficient & \\
\hline$a_{2,1,(p), m}$ & $\begin{array}{l}\alpha_{p}(C h 0 S h 0+C h 1 S h 1)+\beta_{p} A S h 1 C h 1+\gamma_{p} B S h 1 C h 1+\delta_{p} G S h 1 C h 1 \\
+\sum_{i_{p}=1}^{N_{p}}\left(\cosh \left(a_{p, m}, x_{p, i_{p}}\right) \sinh \left(a_{p, m}, x_{p, i_{p}}\right)\right)\end{array}$ \\
\hline$a_{2,2,(p), m}$ & $\alpha_{p}\left(S h 0^{2}+S h 1^{2}\right)+\beta_{p} A C h 1^{2}+\gamma_{p} B S h 1^{2}+\delta_{p} G C h 1^{2}+\sum_{i_{p}=1}^{N_{p}}\left(\sinh \left(a_{p, m}, x_{p, i_{p}}\right)\right)^{2}$ \\
\hline$a_{2,3,(p), m}$ & $\begin{array}{l}\alpha_{p}(C 0 S h 0+C 1 S h 1)-\beta_{p} A S 1 C h 1-\gamma_{p} B C 1 S h 1+\delta_{p} G S 1 C h 1 \\
+\sum_{i_{p}=1}^{N_{p}}\left(\cos \left(a_{p, m}, x_{p, i_{p}}\right) \sinh \left(a_{p, m}, x_{p, i_{p}}\right)\right)\end{array}$ \\
\hline$a_{2,4,(p), m}$ & $\begin{array}{l}\alpha_{p}(S 0 S h 0+S 1 S h 1)+\beta_{p} A C 1 C h 1-\gamma_{p} B S 1 S h 1+\delta_{p} G C 1 C h 1 \\
+\sum_{i_{p}=1}^{N_{p}}\left(\sin \left(a_{p, m}, x_{p, i_{p}}\right) \sinh \left(a_{p, m}, x_{p, i_{p}}\right)\right)\end{array}$ \\
\hline \multicolumn{2}{|c|}{ If $p<N_{p}$ then } \\
\hline$a_{2,5,(p), m}$ & $-\gamma_{p} a_{p, m}^{2} a_{p+1, m}^{2} S h 1$ \\
\hline$a_{2,6,(p), m}$ & $-\beta_{p} a_{p, m} a_{p+1, m}-\delta_{p} a_{p, m}^{3} a_{p+1, m}^{3} C h 1$ \\
\hline$a_{2,7,(p), m}$ & $+\gamma_{p} a_{p, m}^{2} a_{p+1, m}^{2} S h 1$ \\
\hline$a_{2,8,(p), m}$ & $-\beta_{p} a_{p, m} a_{p+1, m}+\delta_{p} a_{p, m}^{3} a_{p+1, m}^{3} C h 1$ \\
\hline
\end{tabular}

Table A4. Coefficients $a_{3, l,(p), m}, l=1, \ldots, 8$, and $p=1,2, \ldots, N_{p}$.

\begin{tabular}{|c|c|}
\hline Coefficient & \\
\hline$a_{3,1,(p), m}$ & $\begin{array}{l}\alpha_{p}(C h 0 C 0+C h 1 C 1)-\beta_{p} A S h 1 S 1-\gamma_{p} B C h 1 C 1+\delta_{p} G S h 1 S 1 \\
+\sum_{i_{p}=1}^{N_{p}}\left(\cosh \left(a_{p, m}, x_{p, i_{p}}\right) \cos \left(a_{p, m}, x_{p, i_{p}}\right)\right)\end{array}$ \\
\hline$a_{3,2,(p), m}$ & $\begin{array}{l}\alpha_{p}(C 0 S h 0+C 1 S h 1)-\beta_{p} A S 1 C h 1-\gamma_{p} B C 1 S h 1+\delta_{p} G S 1 C h 1 \\
+\sum_{i_{p}=1}^{N_{p}}\left(\sinh \left(a_{p, m}, x_{p, i_{p}}\right) \cos \left(a_{p, m}, x_{p, i_{p}}\right)\right)\end{array}$ \\
\hline$a_{3,3,(p), m}$ & $\alpha_{p}\left(C h 0^{2}+C h 1^{2}\right)+\beta_{p} A S 1^{2}+\gamma_{p} B C 1^{2}+\delta_{p} G S 1^{2}+\sum_{i_{p}=1}^{N_{p}}\left(\cos \left(a_{p, m}, x_{p, i_{p}}\right)\right)^{2}$ \\
\hline$a_{3,4,(p), m}$ & $\begin{array}{l}\alpha_{p}(S 0 C 0+S 1 C 1)-\beta_{p} A C 1 S 1+\gamma_{p} B S 1 C 1+\delta_{p} G C 1 S 1 \\
+\sum_{i_{p}=1}^{N_{p}}\left(\sin \left(a_{p, m}, x_{p, i_{p}}\right) \cos \left(a_{p, m}, x_{p, i_{p}}\right)\right)\end{array}$ \\
\hline \multicolumn{2}{|c|}{ If $p<N_{p}$ then } \\
\hline$a_{3,5,(p), m}$ & $+\gamma_{p} a_{p, m}^{2} a_{p+1, m}^{2} C 1$ \\
\hline$a_{3,6,(p), m}$ & $+\beta_{p} a_{p, m} a_{p+1, m}+\delta_{p} a_{p, m}^{3} a_{p+1, m}^{3} S 1$ \\
\hline$a_{3,7,(p), m}$ & $+\gamma_{p} a_{p, m}^{2} a_{p+1, m}^{2} C 1$ \\
\hline$a_{3,8,(p), m}$ & $+\beta_{p} a_{p, m} a_{p+1, m}-\delta_{p} a_{p, m}^{3} a_{p+1, m}^{3} S 1$ \\
\hline
\end{tabular}


Table A5. Coefficients $a_{4, l,(p), m}, l=1, \ldots, 8$, and $p=1,2, \ldots, N_{p}$.

\begin{tabular}{|c|c|}
\hline Coefficient & \\
\hline$a_{4,1,(p), m}$ & $\begin{array}{l}\alpha_{p}(C h 0 S 0+C h 1 S 1)+\beta_{p} A S h 1 C 1-\gamma_{p} B C h 1 S 1+\delta_{p} G S h 1 C 1 \\
+\sum_{i_{p}=1}^{N_{p}}\left(\cosh \left(a_{p, m}, x_{p, i_{p}}\right) \sin \left(a_{p, m}, x_{p, i_{p}}\right)\right)\end{array}$ \\
\hline$a_{4,2,(p), m}$ & $\begin{array}{l}\alpha_{p}(S h 0 S 0+S h 1 S 1)+\beta_{p} A C h 1 C 1-\gamma_{p} B S h 1 S 1+\delta_{p} G C h 1 C 1 \\
+\sum_{i_{p}=1}^{N_{p}}\left(\sinh \left(a_{p, m}, x_{p, i_{p}}\right) \sin \left(a_{p, m}, x_{p, i_{p}}\right)\right)\end{array}$ \\
\hline$a_{4,3,(p), m}$ & $\begin{array}{l}\alpha_{p}(C 0 S 0+C 1 S 1)-\beta_{p} A S 1 C 1+\gamma_{p} B C 1 S 1+\delta_{p} G S 1 C 1 \\
+\sum_{i_{p}=1}^{N_{p}}\left(\cos \left(a_{p, m}, x_{p, i_{p}}\right) \sin \left(a_{p, m}, x_{p, i_{p}}\right)\right)\end{array}$ \\
\hline$a_{4,4,(p), m}$ & $\begin{array}{l}\alpha_{p}\left(S 0^{2}+S 1^{2}\right)+\beta_{p} A C 1^{2}+\gamma_{p} B S 1^{2}+\delta_{p} G C 1^{2} \\
+\sum_{i_{p}=1}^{N_{p}}\left(\sin \left(a_{p, m}, x_{p, i_{p}}\right)\right)^{2}\end{array}$ \\
\hline \multicolumn{2}{|c|}{ If $p<N_{p}$ then } \\
\hline$a_{4,5,(p), m}$ & $+\gamma_{p} a_{p, m}^{2} a_{p+1, m}^{2} S 1$ \\
\hline$a_{4,6,(p), m}$ & $-\beta_{p} a_{p, m} a_{p+1, m}-\delta_{p} a_{p, m}^{3} a_{p+1, m}^{3} C 1$ \\
\hline$a_{4,7,(p), m}$ & $-\gamma_{p} a_{p, m}^{2} a_{p+1, m}^{2} S 1$ \\
\hline$a_{4,8,(p), m}$ & $-\beta_{p} a_{p, m} a_{p+1, m}+\delta_{p} a_{p, m}^{3} a_{p+1, m}^{3} C 1$ \\
\hline
\end{tabular}

Received: 23 January 2017

Revised: 20 May 2017

Re-revised: 4 July 2017

Accepted: 16 July 2017 